\title{
Association of Hardiness and Social Support with Posttraumatic Growth Following Athletic Injuries
}

\author{
Takaharu Nakamura ${ }^{1}$, and Hironobu Tsuchiya ${ }^{2}$ \\ ${ }^{1}$ Department of Psychology, Kobe Gakuin University, 518, Arise, Ikawadani-cho, Kobe, Nishi-ku, Kobe-shi, Hyogo 651-2180, JAPAN \\ nakamura@psy.kobegakuin.ac.jp \\ ${ }^{2}$ Department of Health and Sport Management, Osaka University of Health and Sport Sciences \\ 1-1 Asashirodai, Kumatori-cho, Sennan-gun, Osaka 590-0496 JAPAN \\ [Received March 29, 2019; Accepted February 11, 2020; Published online March 13, 2020]
}

\begin{abstract}
Posttraumatic growth (PTG) is viewed as a positive psychological change resulting from the struggle with trauma. This study was designed to prospective examine the association of Hardiness and Social Support with PTG following athletic injuries. University student-athletes $(N=235)$ completed a baseline survey that included questions about socio-demographics, and the Japanese version of the Hardiness Scale for University Student at Time 1 (T1). The same athletes completed a second survey (T2) that included questions about experiences of sports-related injuries, the assessment of Subjective Units of Distress (SUDs), and responding to the Japanese version of the Social Support Scales for University Students, 14 months after T1. Four months later (T3), 209 of the same athletes completed the PTG Scale After an Athletic Injury (PTGS-AI). Data of 92 participants that met the screening criteria were analyzed using multiple regression analysis. The results indicated that appraisal support was the only factor that significantly predicted PTG $(\beta=.48, p<.01)$ after adjusting for potential covariates such as age and gender. Moreover, hierarchical regression analysis indicated that the association of hardiness and appraisal support with PTG was different for different PTG subscales. The interaction between hardiness and appraisal support was significant for Relating to Team Members $(F(1,87)=3.12, p<.05)$ and Psychological Strength as an Athlete $(F(1,87)=5.02$, $p<.01)$. These findings suggest that developing an environment in which athletes receive appraisal support could contribute to their psychological growth.
\end{abstract}

Key words: prospective study, pretrauma factor

\section{Introduction}

Athletic injuries are significant setbacks encountered in sports medicine and sports psychology. Injuries have central effects that cause problems related to physical strength (Nakamura and Araki, 2016) and peripheral effects at the psychological level, including doubts concerning the ability to continue with the sport, and the possible loss of a future role and the position in the team (Tatsumi, 2013). Previous research has indicated that emotional distress in injured athletes includes anxiety, tension, and depression (Pearson and Jones, 1992).

One concept gaining increased attention in sports psychology research is growth following an acute sport-related injury (e.g., Udry et al., 1997; Wadey et al., 2011, Nakamura et al., 2018). For example, Udry et al. (1997) interviewed 21 injured athletes of a US
Ski Team. They reported that 20 of the 21 skiers in the team perceived they had experienced StressRelated Growth benefits across three dimensions: personal growth, psychological performance enhancements, and physical and technical development. The skiers reported that they not only became physically stronger but learned more about their body, its functions, and how it responds to training. Interestingly, such findings, which demonstrated how the process of recovery from injury might generate unique growth experiences, have not been reported by other researchers investigating growth following stressful or traumatic events (cf. Calhoun and Tedeschi, 1999).

In the last decade, research in the field of trauma has shifted its focus from trauma-related pathologies such as posttraumatic stress disorder (PTSD) to positive adaptation-related outcomes after adversity, such as growth, benefits, and other positive changes. Post- 
traumatic growth (PTG) is viewed as a positive psychological change resulting from a struggle with a highly challenging crisis (Tedeschi and Calhoun, 1996). PTG has been observed among individuals that had suffered various types of trauma, such as cancer (e.g., Nenova et al., 2013), natural disasters (e.g., Xu and Liao, 2011), and physical or sexual assault (e.g., Kleim and Ehlers, 2009). Injured athletes might also experience PTG because athletic injuries can lead to psychological problems in some individuals (e.g., Nakamura et al., 2018). Calhoun and Tedeschi (2004) proposed a model of PTG comprising of several interacting components. However, the differences between athletes that experience PTG after suffering an injury and those that do not experience PTG have not been identified to date. Therefore, this study focused on the concept of hardiness and social support and addressed the issue of PTG after injury.

Hardiness is defined as a personality structure comprising of three general and related dispositions that include commitment, control, and challenge, which functions as a buffer in the face of stressful events and conditions (Kobasa, 1979). Commitment is defined as the tendency of involving the self in life activities through a genuine interest and curiosity about the surrounding world (i.e., activities, objects, other people). Control is defined as the tendency to believe and act as if the self can influence surrounding events through effort. Finally, Challenge is defined as the belief that change, rather than stability, is the standard mode of life and constitutes opportunities that motivate a person for personal growth rather than threaten security.

Joh (2010), reported in a study on hardiness that individuals with a high level of hardiness tend to use problem-focused coping strategies, positive interpretations, and emotional support. The rationale for examining hardiness in the context of athletic injury is that it is conceptualized as transforming debilitating situations into opportunities for personal growth and development (Kobasa, 1979). Salim et al. (2015) recently examined the relationship between the personality trait of hardiness and stress-related growth. In their exploratory study that used a cross-sectional design, 206 athletes that had recently returned to sports following an injury completed measures of hardiness, coping, and stress-related growth. As hypothesized, the results indicated a significant positive relationship between hardiness and stress-related growth, which suggested that athletes with a high level of hardiness, who had experienced an injury that shakes their core beliefs about the self, realized PTG.

Social support is defined as verbal and nonverbal communication between recipients and providers that reduces uncertainty about a situation, the self, others, and relationships. Social support functions to enhance a person's perceptions of personal control in his or her life experiences (Albrecht and Adelman, 1987). Specific studies have demonstrated a positive relationship between social support and treatment adherence, as well as an increased recovery rate in injured athletes (Duda et al., 1989). Moreover, research has consistently demonstrated that social support is vital for reducing stress amongst injured athletes and for improving motivation during rehabilitation (Malinauskas, 2008). Furthermore, Yang et al. (2014) reported that athletes having higher satisfaction with social support from their coaches during recovery were less likely to experience symptoms of depression and anxiety when they return to play. Furthermore, a model developed by Calhoun et al. (2010) also highlights the central role of social support in PTG. The presence of supportive others is considered to be beneficial for the validation of narratives provided by trauma survivors and for offering alternative perspectives that could be integrated into assumptive worlds. A meta-analysis has indicated that social support is moderately associated with PTG (Prati and Pietrantoni, 2009). Therefore, athletes that received social support from team members after they return to play could experience high PTG.

A number of studies have examined whether demographic characteristics (e.g., gender, age; Manne et al., 2004), peritraumatic factors (e.g., peritraumatic distress; Kleim and Ehlers, 2009; Kunst, 2010), and posttrauma factors (e.g., coping style; Kleim and Ehlers, 2009; Widows et al., 2005) affects PTG. However, only a few prospective studies have specifically examined relationships between pre-trauma psychological factors (e.g., psychological distress) and PTG (e.g., Frazier et al., 2009; Moore et al., 2011). Furthermore, according to Calhoun and Tedeschi (1998), the process of PTG involves the interplay between pre and posttrauma psychosocial factors. One of the few studies on this topic has reported that positive cancer-related ruminations mediated the relationship between positive attentional bias and PTG (Chan, Ho, Tedeschi, and Leung, 2011), and adaptive coping mediated the relationship between personality traits such as openness and PTG (Shakespeare-Finch et al., 2005). However, the cross-sectional design of these studies has made the direction of casualty in the observed associ- 
ations unclear. Therefore, the current study was designed to investigate factors predicting PTG prospectively.

Specifically, this 18 -month prospective study investigated the association of hardiness and social support with PTG among injured athletes. In particular, we examined the hypothesis that university studentathletes with a high level of hardiness and perceived social support after an injury experience a high degree of PTG compared to students with a low level of hardiness and perceived social support.

\section{Method}

\subsection{Participants}

University student-athletes $(N=235,168$ men and 67 women, mean age $20.57 \pm 1.01$ years), who were members of five teams in four universities located in the Kinki region of Japan, volunteered to participate in this study. We targeted teams meeting the following criteria: (1) Contact sports, because of the high injury rates in these sports (John, 2014); (2) team sports, including soccer, American football, touch football, field hockey, and Lacrosse; as well as (3) national level sports teams, because Kaplan et al. (2005) reported that differences in the level of competition could have an impact on athletic injury.

\subsection{Procedures}

Approval was obtained from the ethics committee of the first author's affiliated university before conducting the study. We first contacted teams by using e-mail to explain the purpose of this study to the coaches. After that, data were collected in the team meeting rooms at the team training facilities of each university. The voluntarily recruited athletes that had never met the authors were provided with a letter explaining the purpose of the study and informing them that they were free to withdraw from the study at any time without suffering any negative consequences. Coaches were not present for the data collection. No recruits chose to withdraw from the study. All the recruits were asked to complete an informed consent form before participating in the study. Then, the 235 participants completed a baseline survey at Time 1 (T1). After 14 months from T1, 218 athletes (92.7\%) that completed the baseline survey at $\mathrm{T} 1$ completed a second survey at Time 2 (T2); of these, 209 athletes

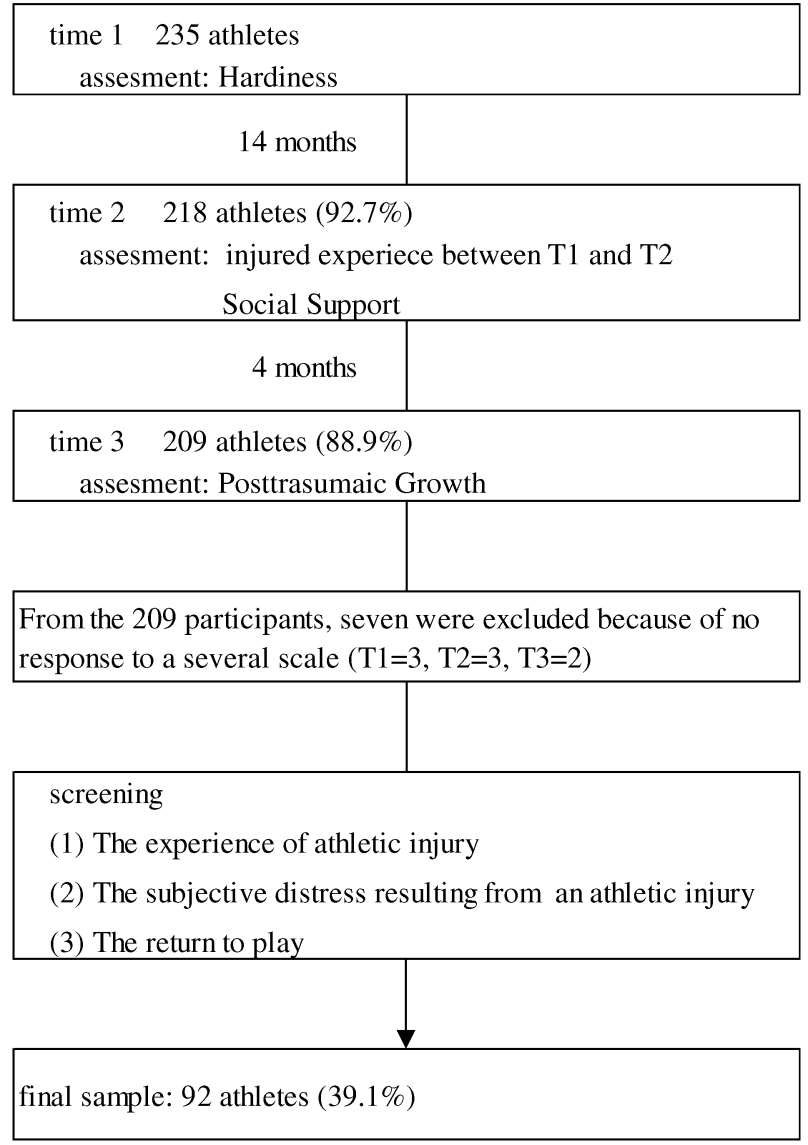

Figure 1 Flowchart of the procedures in this study

$(88.9 \%)$ completed the third survey four months later at Time 3 (T3). Self-administered questionnaires were used in all the surveys. Of the 209 participants that volunteered, seven were excluded because they did not respond to several scales $(\mathrm{T} 1=3, \mathrm{~T} 2=3, \mathrm{~T} 3=2)$. The final response rate was $85.9 \%(N=202)$.

\subsection{Measures}

\subsubsection{Assessment at $\mathrm{T} 1$}

(1) Demographic data

Demographic information including age, gender, type of sport, years of sports experience, and the competitive level of the team was collected. The competitive level of the team was defined as competing in international, national, or regional level games.

\section{(2) Hardiness}

We mainly assessed Hardiness at T1 because the data were collected before an injury had occurred. The Japanese version of the Hardiness Scale for University Students (HS-J: Tada and Hamano, 2001), which consists of 15 items: commitment (5 items), challenge (5 items), and control (5 items) were used to assess har- 
diness. The responses to this scale are made using a 5-point Likert scale ranging from 1 (not at all) to 5 (extremely). Higher scores on this scale indicate a higher level of hardiness in the respondents. The Cronbach's $\alpha$ coefficient of the scale in this study was .80 .

\subsubsection{Assessment at $T 2$}

\section{(1) Sports-related injury experience}

Athletes that had experienced a potential injury between $\mathrm{T} 1$ and $\mathrm{T} 2$ were investigated at $\mathrm{T} 2$. We asked participants to indicate if they had an athletic injury within the last 14 months, which took more than one day to recover. We also requested a medical evaluation, information on the circumstances of the injury, and the number of days taken for recovery. If a participant had experienced multiple athletic injuries, he or she was asked to respond regarding the most serious injury.

(2) Subjective Units of Distress (SUDs: Wolpe, 1973)

SUDs on a scale of 0 (no distress) to 10 (highest distress) points were used to assess the level of psychological distress that the participants experienced because of their athletic injury. Each participant assessed their position on the scale in response to the question, "How distressing was the injury to you?" The subjective distress resulting from athletic injuries differs in different individuals because emotional and affective responses are influenced by an individual's appraisal of a situation. An injured athlete might not experience PTG if he or she does not experience psychological distress as a result of the injury. The validity of SUD's has been confirmed (Tanner, 2012). A number of studies have used SUDs to asses subjective distress (e.g. Takei et al., 2011).

\section{(3) Social support}

The Japanese version of the Social Support Scales for University Students (SSSU-J; Katauke and Onuki, 2014) was used to assess the degree of support that injured athletes received during the post-injury period. This inventory consists of 23 items and three subscales: (1) appraisal support (10 items); (2) information/instrumental support (7 items); and (3) emotional/ companionship support (6 items). Responses to this scale are made using a 4-point scale ranging from 1 (not at all) to 4 (extremely). Higher scores on this scale indicate that more Social Support was received. This scale had sufficient internal reliability for the current sample (Cronbach's alpha for appraisal support $=$ .86 , information/instrumental support $=.92$, and emo- tional/companionship support $=.85$ ).

\subsubsection{Assessment at T3}

\section{(1) PTG following an athletic injury}

We assessed PTG four months after T2 to identify its causal association with Social Support. The PTG Scale After an Athletic Injury (PTGS-AI; Nakamura et al., 2018) was used to assess PTG resulting from the athletic injury described at $\mathrm{T} 2$. This scale consists of 16 items and 4 subscales: (1) 4 items assess Relating to Team Members (RTM); (2) 6 items assess Psychological Strength as an Athlete (PSA); (3) 3 items assess Effort for New Possibilities (ENP); and (4) 3 items assess Better Preparation for Competitions (BPC). Responses to PTGS-AI are made on a 6-point scale ranging from 0 (not at all) to 6 (extremely). The higher was the score on this scale, the higher was the PTG resulting from the injury. The internal reliabilities of the PTGS-AI for the current sample were reasonably good (Cronbach's alpha for RTM was .83, PSA was .83, ENP was .82, and BPC was .68).

\subsection{Data analyses}

\subsubsection{Screening}

(1) The experience of athletic injury

PTG does not occur as a result of mild athletic injuries, but from a significant psychological struggle after a highly stressful life event (Tedeschi and Colhoun, 2004). Therefore, this study, based on previous research (Nakamura et al., 2018), investigated only the participants that experienced an injury that caused them to withdraw from sports for over a day between $\mathrm{T} 1$ and $\mathrm{T} 2$.

(2) The subjective distress resulting from athletic injury

Based on a previous study (Nakamura et al., 2018), only the participants that indicated SUDs between 6-10 points were investigated in this study.

(3) The return to play

A player that had not healed at T3 could potentially still be mentally struggling because of his/her athletic injury. Therefore, only participants that had experienced an athletic injury between $\mathrm{T} 1$ and $\mathrm{T} 2$ that had healed at $\mathrm{T} 3$ were investigated.

\subsubsection{Analysis}

We conducted two primary analyses. First, t-tests for independent samples were conducted to examine possible gender differences in HS-J, SSSU-J, and 
PTGS-AI scores. Then, in Step 1, we conducted hierarchical regression analyses to examine the associations of each social support factor; appraisal, information/instrumental, and emotional/companionship, on each subscale of PTGS-AI. Age, Gender, the type of sport, Competition level, Days for recovery, psychological distress, and hardiness were entered as Confounding Variables. In Step 2, we also tested whether hardiness and social support interacted with any of the PTGS-AI subscales. The interaction term of hardiness and social support was entered into the model as a covariate. We used centered variables to analyze the interaction term of hardiness and social support (Maeda, 2008) to account for possible multicollinearity. Then, we examined possible associations between these variables and each PTGS-AI subscale. Post-hoc analyses were conducted to examine the nature of significant interactions following the procedure recommended by Holmbeck (2002). All the statistical tests were conducted using SPSS statistics version 21.0. We set the level of significance at $5 \%$.

\section{Results}

\subsection{Participating cohort}

Of the participants, 92 met all the screening criteria ( $39 \%$ of the total sample; 64 men and 28 women). The athletes played Soccer $(N=37,40.2 \%)$, American Football $(N=27,29.3 \%)$, Field Hockey $(N=12,13 \%)$, Touch Football $(N=10,10.9 \%)$, or Lacrosse $(N=6$, $6.5 \%)$. The competitive level was National $(N=59$, $64.1 \%)$ or Reginal $(N=33,35.9)$. The median number of days for recovery was 43.5 days $(\mathrm{Q} 1=14$ days, $\mathrm{Q} 3=90$ days, Maximum $=300$ days; Minimum $=3$ days).

\subsection{Gender differences in each measure}

We conducted t-tests for Independent samples to examine possible gender differences in HS-J, SSSU-J, and PTGS-AI scores of the 92 injured athletes. Results indicated no significant gender differences in any of the measures (Table 2). Therefore, we combined the scores of men and women in all the subsequent analyses.

\subsection{The association between social support sub- scale scores and PTG}

As shown in Step 1 of Table 3, all the models were statistically significant $(p<.01 \sim 05)$, with squared

Table 1 Participating cohort meeting all the screening criteria $(N=92)$.

\begin{tabular}{llrr}
\hline \multicolumn{2}{c}{ charateristic } & $N$ & $\%$ \\
\hline gender & male & 64 & 69.6 \\
& female & 28 & 30.4 \\
the type of sport & Soccer & 37 & 40.2 \\
& American Football & 27 & 29.3 \\
& Field Hockey & 12 & 13.0 \\
& Touch Football & 10 & 10.9 \\
the competitive level & Lacrosse & 6 & 6.5 \\
& International & 0 & 0.0 \\
& National & 59 & 64.1 \\
age & Regional & 33 & 35.9 \\
days for recovery & Mean (SD) & $20.39(1.03)$ \\
& Median (Q1, Q3) & $43.5(14,90)$ \\
& Maximum & \multicolumn{2}{c}{300} \\
& Minimum & \multicolumn{2}{c}{3} \\
\hline
\end{tabular}

Table 2 Descriptive statistics and results of t-test on the difference in all variables between males and females $(N=92)$.

\begin{tabular}{|c|c|c|c|c|c|c|c|c|}
\hline & \multicolumn{2}{|c|}{ Total samples } & \multicolumn{2}{|c|}{ Males } & \multicolumn{2}{|c|}{ Females } & \multirow{2}{*}{$t$} & \multirow{2}{*}{$p$} \\
\hline & $M$ & $S D$ & $M$ & $S D$ & $M$ & $S D$ & & \\
\hline Hardiness & 3.85 & 0.60 & 3.81 & 0.48 & 3.94 & 0.81 & -1.06 & .29 \\
\hline Information/Instrumental Support & 2.82 & 0.76 & 2.87 & 0.77 & 2.69 & 0.71 & 1.08 & .28 \\
\hline Emotional/Companionship Support & 2.85 & 0.78 & 2.82 & 0.77 & 2.92 & 0.78 & -0.61 & .55 \\
\hline Appraisal Support & 3.09 & 0.69 & 3.12 & 0.66 & 3.00 & 0.75 & 0.83 & .41 \\
\hline Social Support & 2.91 & 0.67 & 2.93 & 0.67 & 2.84 & 0.68 & 0.62 & .54 \\
\hline Relating to Team Members & 3.36 & 1.30 & 3.24 & 1.30 & 3.63 & 1.29 & -1.33 & .19 \\
\hline Psychological Strength as an Athlete & 3.65 & 0.93 & 3.63 & 0.95 & 3.70 & 0.88 & -0.37 & .71 \\
\hline New Possibilities as an Athlete & 3.56 & 1.32 & 3.60 & 1.33 & 3.47 & 1.30 & 0.42 & .67 \\
\hline Better Preparation on Competition & 3.83 & 1.03 & 3.74 & 1.08 & 4.01 & 0.89 & -1.17 & .24 \\
\hline PTGS-AI & 3.59 & 1.00 & 3.55 & 1.01 & 3.70 & 0.98 & -0.66 & .51 \\
\hline
\end{tabular}

$M=$ mean, $S D=$ Standard deviation 
Table 3 Regression Analysis Predicting Posttraumatic Growth $(N=92)$

\begin{tabular}{|c|c|c|c|c|c|c|c|c|c|}
\hline \multirow{2}{*}{ Outcome } & \multirow{2}{*}{ Independent variables } & \multicolumn{4}{|c|}{ step 1} & \multicolumn{4}{|c|}{ step 2} \\
\hline & & $\beta$ & $p$ & $*$ & VIF & $\beta$ & $p$ & $*$ & VIF \\
\hline \multirow[t]{13}{*}{ Relating to Team Members } & Age & .05 & .67 & & 2.01 & .28 & .13 & & 1.86 \\
\hline & Gender & .00 & .99 & & 7.30 & .11 & .31 & & 8.09 \\
\hline & The type of sport & .03 & .89 & & 7.63 & -.21 & .37 & & 8.83 \\
\hline & Competition level & .07 & .54 & & 1.75 & .12 & .24 & & 1.82 \\
\hline & Days taken for recovery & .14 & .14 & & 1.27 & .12 & .16 & & 1.27 \\
\hline & Psychological distress & -.03 & .82 & & 1.70 & .10 & .38 & & 2.01 \\
\hline & Appraisal & .51 & .00 & $* *$ & 3.15 & .54 & .00 & $* *$ & 3.18 \\
\hline & Information/Instrumental & .03 & .83 & & 3.09 & .03 & .82 & & 3.09 \\
\hline & Emotional/Companionship & .16 & .24 & & 2.78 & .09 & .50 & & 2.88 \\
\hline & Hardiness & .19 & .02 & $*$ & 1.27 & .38 & .04 & $*$ & 3.19 \\
\hline & Hardiness*Appraisal & & & & & .45 & .00 & $* *$ & 4.21 \\
\hline & $R^{2}, F$ & .41 & .01 & $* *$ & 7.36 & .46 & .01 & $* *$ & 7.99 \\
\hline & $\Delta R 2$ & & & & & .05 & .01 & $* *$ & \\
\hline \multirow[t]{13}{*}{ Psychological Strength as an Athlete } & Age & .03 & .89 & & 2.01 & .24 & .09 & & 1.86 \\
\hline & Gender & -.03 & .89 & & 7.30 & .00 & 1.00 & & 8.09 \\
\hline & The type of sport & .20 & .36 & & 7.63 & .17 & .49 & & 8.83 \\
\hline & Competition level & .02 & .82 & & 1.75 & .03 & .76 & & 1.82 \\
\hline & Days taken for recovery & .03 & .74 & & 1.27 & .05 & .65 & & 1.27 \\
\hline & Psychological distress & -.04 & .69 & & 1.70 & -.02 & .84 & & 2.01 \\
\hline & Appraisal & .38 & .01 & $* *$ & 3.15 & .38 & .01 & $* *$ & 3.18 \\
\hline & Information/Instrumental & .13 & .36 & & 3.09 & .13 & .36 & & 3.09 \\
\hline & Emotional/Companionship & .13 & .34 & & 2.78 & .12 & .39 & & 2.88 \\
\hline & Hardiness & .19 & .04 & $*$ & 1.27 & .18 & .04 & $*$ & 3.19 \\
\hline & Hardiness*Appraisal & & & & & .22 & .04 & $*$ & 4.21 \\
\hline & $R^{2}, F$ & .42 & .01 & $* *$ & 7.63 & 0.46 & 0.01 & $* *$ & 7.83 \\
\hline & $\Delta R 2$ & & & & & .04 & .01 & $* *$ & \\
\hline \multirow[t]{13}{*}{ Efforts to New Possibilities } & Age & .12 & .37 & & 2.01 & .08 & .64 & & 1.86 \\
\hline & Gender & -.45 & .07 & & 7.30 & -.45 & .11 & & 8.09 \\
\hline & The type of sport & .13 & .34 & & 7.63 & .14 & .31 & & 8.83 \\
\hline & Competition level & -.18 & .14 & & 1.75 & -.19 & .12 & & 1.82 \\
\hline & Days taken for recovery & .02 & .84 & & 1.27 & .01 & .97 & & 1.27 \\
\hline & Psychological distress & .13 & .27 & & 1.70 & .11 & .39 & & 2.01 \\
\hline & Appraisal & .26 & .04 & $*$ & 3.15 & .26 & .04 & $*$ & 3.18 \\
\hline & Information/Instrumental & .04 & .81 & & 3.09 & .04 & .81 & & 3.09 \\
\hline & Emotional/Companionship & .09 & .53 & & 2.78 & .11 & .49 & & 2.88 \\
\hline & Hardiness & .23 & .02 & $*$ & 1.27 & .24 & .02 & $*$ & 3.19 \\
\hline & Hardiness*Appraisal & & & & & -.07 & .69 & & 4.21 \\
\hline & $R^{2}, F$ & .26 & .01 & $* *$ & 4.21 & .26 & .01 & $* *$ & 4.22 \\
\hline & $\Delta R 2$ & & & & & .01 & .69 & & \\
\hline \multirow[t]{13}{*}{ Better Preparationx on Competition } & Age & .09 & .53 & & 2.01 & .10 & .49 & & 1.86 \\
\hline & Gender & .04 & .87 & & 7.30 & .07 & .78 & & 8.09 \\
\hline & The type of sport & -.09 & .41 & & 7.63 & -.07 & .53 & & 8.83 \\
\hline & Competition level & -.06 & .58 & & 1.75 & -.05 & .64 & & 1.82 \\
\hline & Days taken for recovery & .18 & .06 & & 1.27 & .18 & .06 & & 1.27 \\
\hline & Psychological distress & .17 & .12 & & 1.70 & .19 & .12 & & 2.01 \\
\hline & Appraisal & .59 & .00 & $* *$ & 3.15 & .59 & .00 & $* *$ & 3.18 \\
\hline & Information/Instrumental & -.05 & .72 & & 3.09 & -.05 & .72 & & 3.09 \\
\hline & Emotional/Companionship & -.13 & .36 & & 2.78 & -.14 & .33 & & 2.88 \\
\hline & Hardiness & .21 & .05 & $*$ & 1.27 & .21 & .05 & $*$ & 3.19 \\
\hline & Hardiness*Appraisal & & & & & .06 & .71 & & 4.21 \\
\hline & $R^{2}, F$ & .36 & .01 & $* *$ & 6.16 & .36 & .01 & $* *$ & 5.56 \\
\hline & $\Delta R 2$ & & & & & -.01 & .71 & & \\
\hline
\end{tabular}

** $p<.01, * * p<.05 \quad \beta=$ Standardized Partial Regression Coefficients, VIF = Variance Inflation Factor 
multiple correlations of .26 .42. Results indicated that appraisal and hardiness significantly predicted each subscale of PTGS-AI, whereas information/ instrumental, and emotional/companionship were not significant predictors.

\subsection{The association between hardiness and ap- praisal support with PTG}

As shown in Step 2 of Table 2, hardiness and ap-

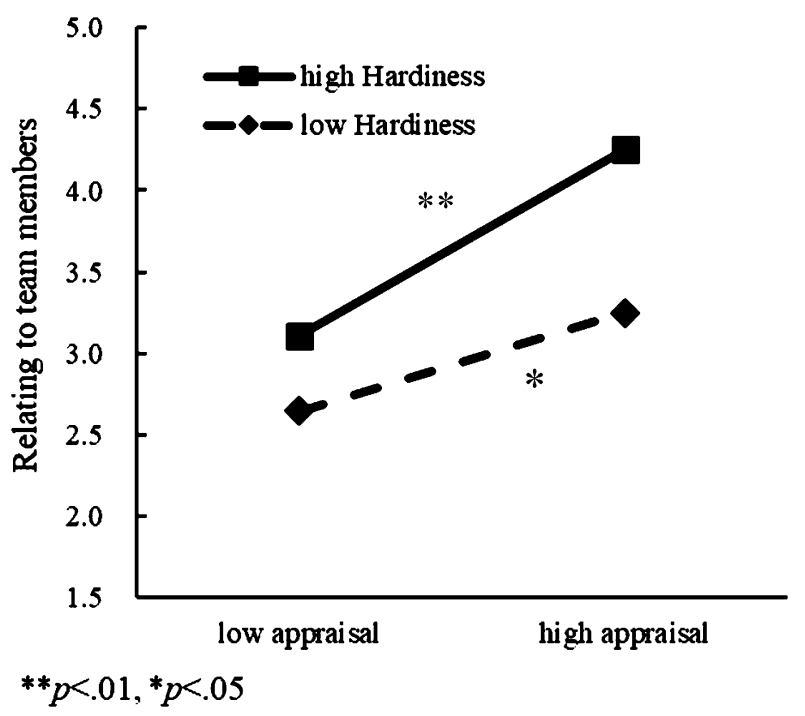

Figure 2 Regression lines of relating to team members on appraisal support as a function of low and high levels of Hardiness

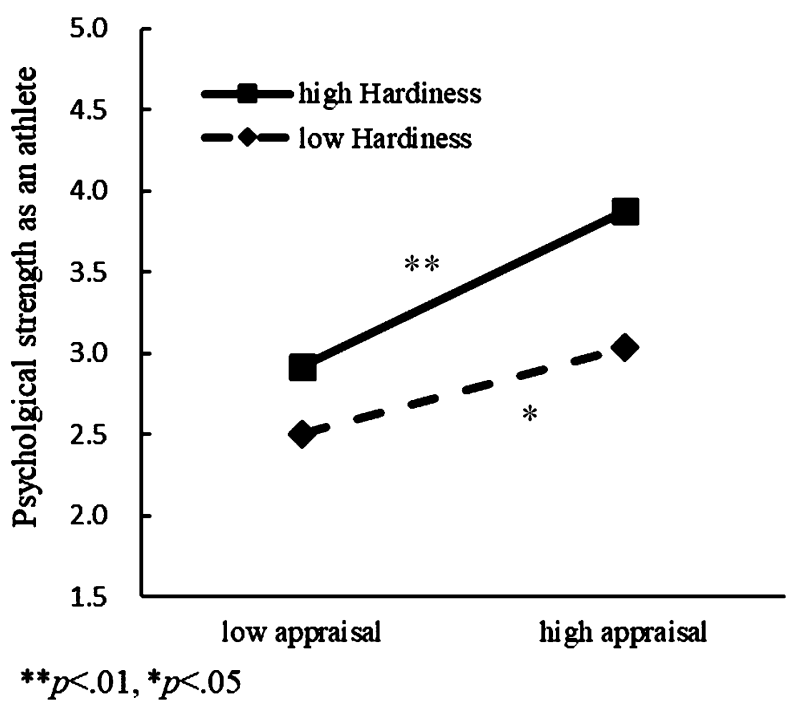

Figure 3 Regression lines of psychological strength as an athlete on appraisal support as a function of low and high levels of Hardiness praisal support affected each PTGS-AI subscale score. Moreover, the interaction term of hardiness and appraisal support was a significant predictor of Relating to Team Members and Psychological Strength as an Athlete as indicated by the increase in the coefficient of determination of $4 \sim 5 \%(p<.01)$.

Post-hoc analyses were conducted to determine the nature of the significant interaction between hardiness and appraisal support with Relating to Team Members and Psychological Strength as an Athlete. As shown in Figure 1, the results of the regression analysis for high hardiness indicated that appraisal support was significantly associated with Relating to Team Members $(\beta=.53, p<.01)$. In contrast, the results of the regression analysis for low hardiness indicated that appraisal support was also significantly associated with Relating to Team Members $(\beta=.27, p<.05)$. Moreover, as shown in Figure 2, the results of the regression analysis for high hardiness indicated that appraisal support was significantly associated with Psychological Strength as an Athlete $(\beta=.45, p<.01)$. In contrast, the results of the regression analysis for low hardiness indicated that appraisal support was also significantly associated with Psychological Strength as an Athlete $(\beta=.24, p<.05)$.

\section{Discussion}

We investigated the association between pre- and post-injury psychological factors and PTG, including hardiness and social support, by using a prospective design. First, we examined the types of social support that were significantly associated with PTGS-AI. Then, we examined the association of hardiness and appraisal support and their interactions with PTGS-AI. Results indicated that appraisal support significantly predicted each PTGS-AI subscale score, whereas information/instrumental and emotional/companionship were not significant predictors. Moreover, there was an interaction between hardiness and appraisal support with Relating to Team Members and Psychological Strength as an Athlete. These results partially supported the hypothesis of this study.

\subsection{The association between social support and PTGS-AI}

The literature suggests that social support is one of the predictors of PTG resulting from several types of 
trauma (Su and Chen, 2015). However, the relationship between social support and PTG after an athletic injury has not been investigated to date. Therefore, the current study investigated the association of different dimensions of support from team members with PTGS-AI. Results indicated that appraisal support was the only significant predictor of PTG and that other types of social support were not significant predictors. Appraisal support is also known as "self-esteem support" or "esteem support" (Wada, 1992), which occurs when expressing positive support for another person and includes confidence and acceptance of the other individual.

Athletes worry about their competitive career during rehabilitation after an injury, and after returning to competition (Tatsumi, 2014). Moreover, some athletes cannot actively compete after an injury (Rottella and Heyman 1986). Athletic injuries also affect psychosocial functioning because of doubts about continuing with the sport, or the possible loss of future roles and the team position (Tatsumi, 2013), which could lead to the collapse of an athlete's identity. Recognition and acceptance from others can restore the mental balance and result in deliberate ruminations regarding returning to play (Tedeschi and Calhoun, 2004). This study indicated that appraisal support could be critical compared to information/instrumental or emotional/ companionship support in promoting PTG after an injury of a team member.

\subsection{Association of hardiness and appraisal support with PTGS-AI}

We also investigated the association of hardiness and appraisal support with PTG using hierarchical regression analyses. The effect of the interaction between hardiness and appraisal support with Relating to Team Members indicated the regulatory effect of appraisal support with High and low hardiness levels. Joh (2010) reported that individuals with high levels of hardiness tended to engage in problem-focused coping and positive interpretations. Therefore, injured athletes with high levels of hardiness might be more likely to take a problem-focused coping style and engage in positive interpretations of events. When this is combined with high perceived social support, athletes are likely to experience Relating to Team Members, which is suggestive of the growth in skills of relating to others (Nakamura et al., 2018). We also found that athletes with low levels of hardiness might experience
Relating to Team Members if they received appraisal support during the period of recovery from an injury.

Results identical to those for Relating to Team Members were observed for Psychological Strength as an Athlete. It has been suggested that athletes with high levels of hardiness are predisposed to experiencing PTG after injuries, and when attempting to cope with injuries. A sense of personal strength, which includes "being strong," and "being more confident," is a hypothetical factor associated with PTG (Taku et al., 2007). PTG includes changes in the self-concept when relating to others, and in the philosophy of life (Tedeschi et al., 1998). Psychological Strength as an Athlete falls into the category of the self-concept, which is conceptually similar to hardiness. Thus, Psychological Strength as an Athlete might be promoted when injured athletes have a high degree of hardiness. The result of the current study indicates that athletes with low hardiness could experience Psychological Strength as an Athlete by received appraisal support. Salim et al. (2017) reported that athletes with low hardiness tended not to disclose their thoughts and feelings to members of their support network because of the emotional climate, personal beliefs, and support exchanges. Katagami and Tsuchiya (2017) suggested that esteem support from teammates positively affected self-confidence. Therefore, athletes with low hardiness in this study might have experienced a change of psychological strength by receiving support from teammates.

Results also indicated that hardiness and appraisal support were independently associated with Effort for New Possibilities, although there was no interaction between these variables. When there is no athletic injury, Effort for New Possibilities represents making new efforts in competitions (Nakamura et al., 2018). Maddi (2004), suggesting that hardiness enhances performance and health despite stressful changes, and increases perceptions and actions consistent with choosing a future orientation. Following an injury, however, athletes with a high degree of hardiness might explore many different paths. Additionally, Hiraki (2006) suggested that social support, including relating to others and new possibilities, were factors that promoted PTG. Therefore, injured athletes might recognize their acceptance by others and focus on what they can accomplish.

Finally, results indicated that appraisal support predicted Better Preparation for Competitions, whereas hardiness did not. Better Preparation for Competitions 
is a type of PTG that is unique to athletic injuries. Nakamura et al. (2018) demonstrated a positive correlation between Better Preparation for Competitions and deliberate rumination, which is an essential factor in Calhoun and Tedeschi's model of PTG. Among stroke survivors, moderate, positive correlations have been demonstrated between PTG, rumination, and social support (Peng and Wan, 2018). Injured athletes receiving appraisal support from team members might become able to ruminate. As a result, they might become better prepared for competitions by improving eating and sleeping habits to prevent injuries.

\subsection{Implication}

The growth following a sport-related injury is gaining increased attention in sport psychology research (Nakamura et al., 2018). In the context of applied sports, injured athletes need not only physical support but also psychological support for injured athletes. However, only a few studies have been conducted on providing psychological support for them. The current study suggests that developing an environment in which athletes receive appraisal support could contribute to their psychological growth. Future research should include more detailed information on adequate support in the rehabilitation process.

\subsection{Limitation}

We have noted certain limitations of this study. Firstly, the sample of this study comprised only of competition team members, and therefore, the results of the study might be valid only for team competitions. A second consideration is that providers of social support were limited. Kataganmi and Tsuchiya (2017) suggested that the effectiveness of received support might depend on the dimensions and providers of support. In the current study, supporters were defined as team members. Team members might also include psychotherapists, such as clinical psychologists. It is suggested that future studies explore the effects of different types of social support providers. Third, it is necessary to use a reliable and valid scale to assess the psychological impact of injuries. SUD measures the subjective intensity of distress experienced by an individual. However, the reliability and validity of SUD are uncertain because it is a response to just one item. Finally, we did not collect data on the ethnicity of the participants. Ethnic diversity is increasing in Japan, and it needs to be considered in future studies.

\section{Conclusion}

This study investigated the association of hardiness and social support with PTG in injured athletes in a longitudinal survey. We found that only appraisal support among social support affected PTG after an athletic injury. Moreover, the association of hardiness and appraisal support with PTG differed depending on the subscale of the PTG such that the interaction of hardiness and appraisal support with Relating to Team Members and Psychological Strength as an Athlete were significant, but not with Effort for New Possibilities or Better Preparation for Competitions. Preventing injuries in athletes is an essential consideration. According to Oka (2000), better control and management of coaches and athletes could decrease the risk of athletic injury. However, not all injuries can be prevented. Nevertheless, the results of this study suggest that developing an environment in which athletes receive appraisal support could contribute to their psychological growth.

\section{References}

Albrecht, T. L. and Adelman, M. B. (1987). Communicating social support. Thousand Oaks, CA, US: Sage Publications, Inc.

Tanner, B. A. (2012). Validity of Global Physical and Emotional SUDS. Appl. Psychophysiology and Biofeedback, 37(1):31-34.

Calhoun, L. G., Cann, A., and Tedeschi, R. G. (2010). The posttraumtic growth model: Sociocultural consideration. In Weiss, T., and Berger, R. (Eds.), Posttraumatic growth and ulturally competent practice: Lessons learned from around the globe. Hoboken, NJ: John Wiley and Sons, Inc. pp.1-14.

Calhoun, L. G. and Tedeschi, R. G. (1999). Facilitating posttraumatic growth: Aclinician's guide. Mahwah, NJ: Erlbaum.

Chan, M. W. C., Ho, S. M. Y., Tedeschi, R. G., and Leung, C. W. L. (2011). The valence of attentional bias and cancer-related rumination in posttraumatic stress and posttraumatic growth among women with breast cancer. Psycho-Oncology, 20, 544552.

Duda, J. T., Smart, A. E., and Tappe, M. K. (1989). Predictors of adherence in the rehabilitation of athletic injuries: An application of personal investment theory. J. Sport Exerc. Psychol., 11:367-381.

Frazier, P., Tennen, H., Gavian, M., Park, C., Tomich, P., and Tashiro, T. (2009). Does self-reported posttraumatic growth reflect genuine positive change? Psychological Science, 20, 912919.

Hiraki, K. (2006). What contributes to Posttraumatic growth: focus on the growth process. Bull. Fac. Contemp. Socail Studies, Nagasaki Wesleyan Univ., 4:75-84. (in Japanese).

Holmbeck, G. N. (2002). Post-hoc probing of significant moderational and mediational effects in studies of pediatric populations. J. Pediatr. Psychol., 27:87-96. 
Joh, Y. (2010). Relationship of Hardiness to Stress-coping Strategies and Life Events in University Students. Bull. Liv. Sci., Bunkyo Univ., 32:37-47.

John, T. P. (2014). NCAA sports medicine handbooks. Overland Park, KS: National Collegiate Athletic Association, 25-26.

Kaplan, L. D., Flanigan, D. C., Norwig, J., Jost, P., and Bladly, J. (2005). Prevalence and variance of shoulder injuries in elite collegiate football player. Am. J. Sports Med, 33:1142-1146.

Katagami, E. and Tsuchiya, H. (2017). Effects of Received Social Support on Athletes' psychological well-being. Int. J. Sport health Sci., 15:72-80. (in Japanese).

Katauke, Y. and Onuki, N. (2014). Development and Validation of Social Support Scales for Japanese College Students: Including Appraisal Support. J.psychol. Rissho Univ., 5:37-46. (in Japanese).

Kleim, B. and Ehlers, A. (2009). Evidence for a Curvilinear Relationship between Post-Traumatic Growth and Post-Trauma Depression and PTSD in Assault Survivors. J. Trauma. Stress, 22, 45-52.

Kobasa, S. C. (1979). Stressful life events, personality and health: An inquiry into hardiness. J. Pers. Soc. Psychol., 37:1-11.

Kunst, M. J. (2010). Peritraumatic distress, posttraumatic stress disorder symptoms, and posttraumatic growth in victims of violence. J. Trauma. Stress, 23, 514-518.

Maddi, S. R. (2004). Hardiness: An Operationalization of Existential Courage. J. Human. Psychol., 44:279-298.

Maeda, K. (2008). Applications of multiple regression analysis: analysis including interaction term and control variables. Bulletin of Hijiyama University Junior College (43), 69-73. (in Japanese).

Malinauskas, R. (2008). College athlete's perceptions of social support provided by their head coach before injury and after. J. Sports Med. Phys. Fitn., 48:107-112.

Manne, S., Ostroff, J., Winkel, G., Goldstein, L., Fox, K., and Grana, G. (2004). Posttraumatic growth after breast cancer: Patient, partner, and couple perspectives. Psychosom. Med., 66, 442-454.

Nakamura, T. and Araki, M. (2016). The application of "The five stages of grief" to acceptance of athletic injury: Case studies with qualitative approach. Osaka Res. J. Phys. Edu., 54:31-40. (in Japanese).

Nakamura, T., Tsuchiya, H., and Taku, K. (2018). The Features of Post-traumatic Growth After Athletic Injury. Japan J. Phys. Educ. Hlth. Sport Sci., 63:291-304. (in Japanese).

Nenova, M., DuHamel, K., Zemon, V., Rini, C., and Redd, W. H. (2013). Posttraumatic growth, social support, and social constraint in hematopoietic stem cell transplant survivors. Psycho-Oncology, 22:195-202.

Oka, K. and Ueda, M. (Eds.) (2000). Handbook of Sport Psychology. JITSUMUKYOIKU-SHUPPAN Co., Ltd: Tkyo, pp.399400. (in Japanese).

Pearson, L. and Jones, G. (1992). Emotional Effects of Sports Injuries: Implications for Physiotherapists. Physiother., 78:762770.

Peng, Z. Y. and Wan, L. H. (2018). Posttraumatic Growth of Stroke Survivors and Its Correlation with Rumination and Social Support. J. Neurosci. Nurs., 50:252-257.

Prati, G. and Pietrantoni, L. (2009). Optimism, Social Support and Coping Strategies as Factors Contributing to Posttraumatic Growth: A Meta-Analysis. J. Loss Trauma, 14:364-388.

Rotella, R. J. and Heyman, S. R. (1986). "Stress, injury, and the psychological rehabilitation of athletes". In Applied sport psychology, Edited by Williams, J. M. 343-364. Mountain View,
CA: Mayfield Publishing Company.

Salim, J., Wadey, R., and Diss, C. (2015). Examining the relationship between hardiness and perceived stress-related growth in a sport injury context. Psychol. Sport Exerc., 19:10-17.

Shakespeare-Finch, J., Gow, K., and Smith, S. (2005). Personality, coping and posttraumatic growth in emergency ambulance personnel. Traumatology, 11, 325-334.

Su, Y. J. and Chen, S. H. (2015). Emerging Posttraumatic Growth: A Prospective Study with Pre- and Posttrauma Psychological Predictors. Psychol. Trauma, 7:103-111.

Tada, S. and Hamano, K. (2003). The Development of a Hardiness Scale: Its Reliability and Validity. Notre Dame Seishin Univ. Kiyo. Stud. Fam. life Manag. child welfare, food nutrition, 27:56-62. (in Japanese).

Taku, K., Calhoun, L. G., Tedeschi, R. G., Gil-Rivas, V., Kilmer, R. P. and Cann, A. (2007). Examining posttraumatic growth among Japanese university students. Anxiety, Stress, Coping, 20:353-367.

Tatsumi, T. (2013). Development of Athletic Injury Psychological Acceptance Scale. J. Phys. Ther. Sci., 25:545-552.

Tatsumi, T. (2014). Relationship between adaptation after returning to competition and psycho-behavioral attitudes during injury rehabilitation. J. Phys. Ther. Sci., 26:1813-1823.

Tedeschi, R. G. and Calhoun, L. G. (1996). The Posttraumatic Growth Inventory: measuring the positive legacy of trauma. J. Trauma Stress. 9:455-471.

Tedeschi, R. G. and Calhoun, L. G. (2004). Posttraumatic Growth: Conceptual Foundations and Empirical Evidence. Psychol. Inq., 15:1-18.

Tedeschi, R. G., Park, C. L., and Calhoun, L. G. (Eds.). (1998). Posttraumatic growth: Positive change in the aftermath of crisis. Mahwah, NJ: Erlbaum.

Udry, E., Gould, S., Bridges, D., and Beck, L. (1997). Down but not out: Athlete responses to season-ending injuries. J. Sport Exerc. Psychol., 19, 229-248.

Wada, M. (1992). Effects of social supports on freshmen's psychological factor. Jap. J. Edu. Psychol., 40:386-393. (in Japanese).

Wadey, R., Evans, L., Evans, K., and Mitchell, I. (2011). Perceived benefits following sport injury: A qualitative examination of their antecedents and underlying mechanisms. J. Applied Sport Psychol., 23, 142-158.

Widows, M. R., Jacobsen, P. B., Booth-Jones, M., and Fields, K. K. (2005). Predictors of posttraumatic growth following bone marrow transplantation for cancer. Health Psychol., 24, 266-273.

Wolpe, J. (1973). The practice of therapy (2nd Ed). Pergamon Press, New York.

$\mathrm{Xu}$, J. and Liao, Q. (2011). Prevalence and predictors of posttraumatic growth among adult survivors one year following 2008 Sichuan earthquake. J. Affect. Disord., 133:274-280.

Yang, J., Schaefer, J. T., Zhang, N., Covassin, T., Ding, K., and Heiden, E. (2014). Social Support from the Athletic Trainer and Symptoms of Depression and Anxiety at Return to Play. J. Athl. Train., 49:773-779. 


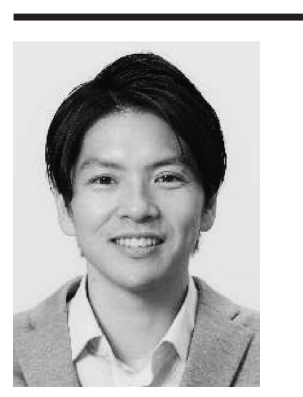

\section{Name:}

Takaharu Nakamura

Affiliation:

Department of Psychology, Kobe Gakuin University, 518, Arise, Ikawadani-cho, Kobe, Nishi-ku, Kobe-shi, Hyogo 6512180, JAPAN

\section{Address:}

518, Arise, Ikawadani-cho, Kobe, Nishi-ku, Kobe-shi, Hyogo 6512180, JAPAN

Brief Biographical History:

2014 Ba. (Sport Sciences), Tenri University

2016 M. A. (Sport Sciences), Osaka University of Health and Sport Sciences

2019 Doctorial program completed with expulsion, Osaka University of Health and Sport Sciences

2018- Lecturer, Department of Psychology, Kobe Gakuin University

\section{Main works:}

-Nakamura, T., Tsuchiya, H., and Taku, K. (2018). The Features of Post-traumatic Growth After Athletic Injury. Japan J. Phys. Educ. Hlth. Sport Sci., 63:291-304.

Membership in Learned Societies:

- Japanese Society of Physical Education, Health and Sport Sciences

-The Japanese Psychological Association

- Japanese Society of Sport Psychology 